\title{
Enterprise Interoperability for Global Collaborative Networks through Cloud Knowledge-Oriented Collaboration System
}

\author{
Hsiao-Kang Lin*, Chun-I Chen and N. C. Wei
}

Department of Industrial Management, I-Shou University, Kaohsiung, Taiwan

\begin{abstract}
New opportunities for global enterprise collaboration are leading into an Information and Communications Technology (ICT) era of network ubiquity through the development of new cooperative cyber-processes, which will provide the ability to deliver real-time integration capabilities. This move is supported by interconnected clouds computing with the idea of extending interoperable software and hardware environments from a physical to a virtual infrastructure. Whilst the Internet and cloud technology are indispensable for the operation and collaboration of modern enterprises, they remain nevertheless, at the level of cloud-enabled databases and IT software integration. They fall far short of the knowledge that is relevant for collaborative creation and operation. This paper aims to introduce and develop the concept of a Cloud Knowledge-Oriented Collaboration Systems (cKOCS), which combines cloud computing, the knowledge community, self-learning knowledge and rule-based moderator technology for global collaborative networks. The cloud knowledgeoriented collaboration system must facilitate the effective and efficient transmission of knowledge with the right expertise and those that enable connections with the right information, enable ad-hoc collaboration, provide a platform to establish real-time notification and awareness in collaborative situations and support collaborative enterprises decision making among participations.
\end{abstract}

Keywords: Enterprise interoperability; Global collaborative networks; Cloud computing; Knowledge-oriented collaboration system

\section{Introduction}

Recent trends towards better development of more effective strategic-alliances have led to Global Collaborative Networks (GCNs) being widely applied as part of many enterprises' business policy to gain competitive advantage, to exploit market opportunities and to provide agility to cope with turbulent market conditions, etc. The success of GCNs' operations is vitally dependent on close collaboration and interoperability between aligned organisations and enterprises. According to Li et al. [1] described that Enterprise Interoperability (EI) aims to improve the manner in which enterprises, assisted by means of Information and Communications Technologies (ICT), interoperate with other enterprises, organisations, or with other business units of the same enterprise, in order to conduct their business. ICT are indispensable to modern enterprise's operation and collaboration. Furthermore cloud computing comes into a way to increase capacity or add capabilities without investing in new infrastructure or licensing new software. Cooperation in mostly heterogeneous cloud environments needs to take up diverse processes, disparate ICT systems and un-normalized communication structures in order to plug-and-play participation of companies into multiple Virtual Enterprises (VEs). A significant number of research and technology have integrated computation, networking, mass storage and various cloud infrastructure (e.g. Amazon Web Services (AWS), Google App Engine, Windows ${ }^{\circledR}$ Azure $^{\mathrm{nm}}$, OpenStack ...etc.), systems and tools (e.g. SAP Cloud Applications Studio, IBM WebSphere Cloud...etc.) in an excellent position in this respect, as shown in Figure 1.

While the above research and technology makes enterprise collaboration system necessary, they remain nevertheless at the level of cloud-enabled databases and IT software integration. They fall far short of knowledge relevant to collaborative creation and operation. Furthermore, the need for intelligent collaboration to promote both necessitates and facilitates collaboration. This is not currently met by existing enterprise collaboration system.

In terms of the validity of knowledge interoperability between current enterprise collaboration systems, some specific challenges facing today's collaborations as identified by Cross et al.; Popplewell et al. $[2,3]$ as following:

Untapped Expertise: Considerable innovation and productivity may be lost owing to the failure to harness unidentified or untapped expertise. Frustratingly, everyone involved in the collaboration understands that the skills are almost certainly already available from within the existing community, the challenge is how to identify and make use of this expertise at the time it is required.

$>$ Mismanaged information: Unfamiliarity with and unawareness of each other's work may easily lead to duplication of work, inefficiencies, wrong estimations, etc. Even worse, "uncontrolled" and unsystematic collaboration about complex issues may be subject to misunderstandings or wrong decisions due to missing knowledge about the business partner; it also exposes the risk of unaware and accidental disclosure of corporate secrets.

$>$ Misunderstood Knowledge: Communication, knowledge sharing and awareness of available expertise are complex issues for any multi-disciplined team. Furthermore, misunderstood knowledge exchange may cause direct problems such as mistakes or confidentiality problems.

*Corresponding author: Hsiao-Kang Lin, Department of Industrial Management, I-Shou University, Kaohsiung, Taiwan, Tel: 8867657 7711; E-mail: hklin@isu.edu.tw

Received April 07, 2015; Accepted May 08, 2015; Published May 18, 2015

Citation: Lin HK, Chen Cl, Wei NC (2015) Enterprise Interoperability for Global Collaborative Networks through Cloud Knowledge-Oriented Collaboration System. J Glob Econ 3: 140. doi:10.4172/2375-4389.1000140

Copyright: (c) 2015 Lin HK, et al. This is an open-access article distributed under the terms of the Creative Commons Attribution License, which permits unrestricted use, distribution, and reproduction in any medium, provided the original author and source are credited. 
Citation: Lin HK, Chen Cl, Wei NC (2015) Enterprise Interoperability for Global Collaborative Networks through Cloud Knowledge-Oriented Collaboration System. J Glob Econ 3: 140. doi:10.4172/2375-4389.1000140

Unable to Learn: Knowledge should be effectively reused and exploited [3,4]. Collaboration opportunities may be missed because of lost opportunities to learn or adapt from past collaborations.

\section{Concept of the Knowledge-Oriented Collaboration Systems}

In response to the challenges of knowledge interoperability, the next generation of knowledge-oriented collaboration system should be able to inter-connect with the right expertise quickly and easily, provide interoperability with the right information (including capture, storage, search, discovery and re-use of content / context) between heterogeneous environments, and enable opportunities for the learning and creation of new knowledge, as following.

\section{Inter-connections with the right expertise through knowledge community}

Studies $[5,6]$ show that human-related issues in the form of social ties and knowledge sharing were reported as keys to successful cooperation within collaborative networks. Several theories and proposals on "Community of Practice" (CoP) and knowledge management $[7,8]$ advocated the concept of $\mathrm{CoP}$ has become associated with finding, sharing, transferring, and archiving knowledge, as well as making explicit "expertise", or tacit knowledge. In today's global organizations and emerging forms of virtual organizations, a Virtual CoP scenario is spurring interest through the building of an internet-based CoP in a virtual team [9]. Recently the rise in popularity of social networking service, by allowing members to express their interests and expertise on the Internet and share content with others and to provide relevant and accurate knowledge as and when needed. These sites enable business partner to assemble into communities and thus improve their effectiveness and making the right connections quickly and easily. Therefore, knowledge community will be easily accomplished by setting of transferring knowledge quickly and efficiently to increase the knowledge sharing speed and allow multi-discipline project team getting the right information at right time, and then make right decision to do things right.

\section{Interoperability with the right information through cloud- enabled IT integration}

The importance of understanding knowledge sharing with right information has become even more evident with the rise of digital knowledge networks. Researchers of many disciplines have studied ontology and semantic web technologies to enable interoperability of disparate information systems for knowledge processes [10,11]. However, where fully integrated approaches have been developed, problems reside in the scalability of the solution. The complexity and cost of the solution is prohibitive for suppliers and sub-suppliers, typically SMEs who don't have the critical competences to develop, customise and implement integrated IT systems for manufacturing. SMEs require low-cost solutions, modular system architectures and interface standards. Above all standardisation will be key along with flexibility in the manufacturing process and integration architecture based on a "cloud" of databases inside the whole supply chain.

\section{Evolution and reuse knowledge through knowledge discovery and self-learning}

Knowledge management is an approach not only to detective, capture, organizes store, but also to share, apply and re-use knowledge within the business context and to the organisation's benefit. The paramount value added knowledge to the success of the business is to reuse of knowledge and learning from one's own experience, best practices of others and past history [12]. Figure 2 illustrates the overall idea of the next generation of knowledge-oriented collaboration system for successful global business to promote and facilitate knowledge creation and operation.

\section{Architecture of the Cloud Knowledge-Oriented Collaboration Systems}

The next generation of knowledge-oriented collaboration system must facilitate the effective and efficiency transmission of knowledge with the right expertise and those that enable connections with the right information in a cloud-based interoperability, by providing a platform to establish real-time notification and awareness in collaborative situations, enable ad-hoc collaboration (non-routine request processing) and support collaborative enterprises decision making among parties. This paper aims to introduce and develop the concept of a Cloud Knowledge-Oriented Collaboration Systems (cKOCS), which combines cloud computing, the knowledge community, rule-based moderator technology and self-learning knowledge to enhance the

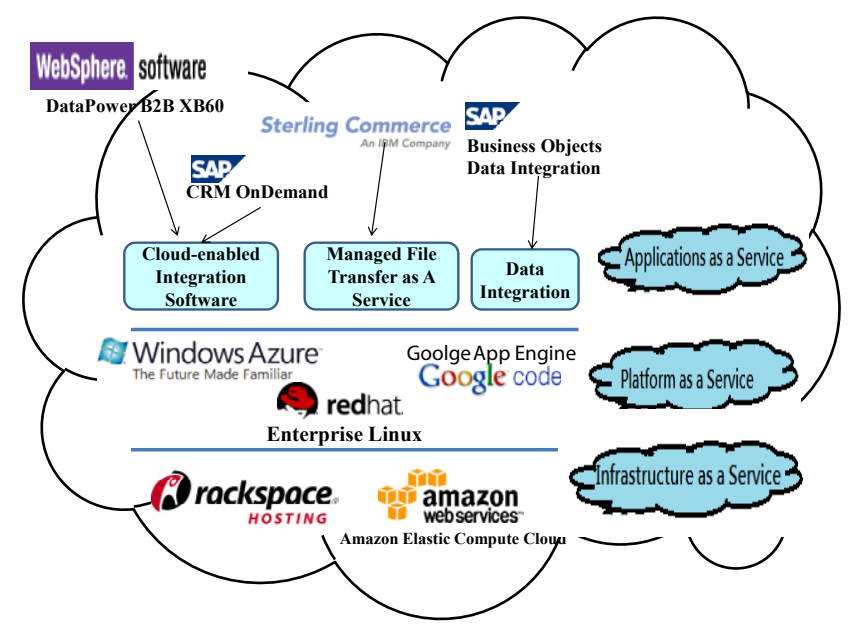

Figure 1: The current cloud infrastructure, systems and tools

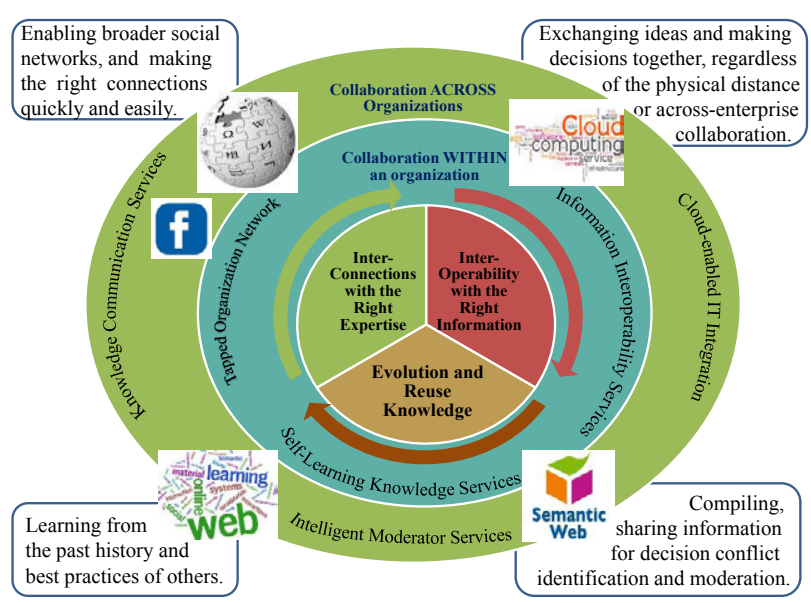

Figure 2: The vision of next generation of knowledge-oriented collaboration system. 
existing enterprise collaboration infrastructure for Global Collaborative Networks (GCNs). The work in cKOCS is organized as following:

\section{Conceptual architecture}

We first illustrated the conceptual and software structure of the cKOCS system. All necessary interfaces is identified on a high level and then specified on an implementation level. Figure 3 presents a high level representation of the cKOCS architecture, which can be structured into four modules: cKOCS Portal, Partner Registry Service, Service Bus, and Unified Service Agent (USA). The cKOCS supports cloud computing through the Windows ${ }^{\oplus}$ Azure ${ }^{\mathrm{m} w}$ platform. Azure offers several ways to build cloud application: Azure Websites, Cloud Services, and Virtual Machines. The basic structure and functions of each module are discussed in the following sections (Figure 3).

cKOCS portal: is a collaboration platform providing a user interface and allowing users to access to the all services provided by the cKOCS. The web portal built using Azure Websites tools, which provide web frontend user interface to the cloud.

$>$ Partner registry service: The collaborative enterprises would need to subscribe to the Partner Registry Service and become a formal cKOCS member, who is also able to access cKOCS various services according their authorization level.

$>$ USA services: are the components that deliver the Knowledge Community Service, Cloud Metadata Service, Knowledge Learning Services and Collaboration Moderator Service. The functions of each component will be further presented in next sections.

$>$ Services bus: the components of USA Services are exposed to the Services Bus that ensures interoperability between them and can be invoked by cKOCS member.

\section{cKOCS USA services}

The cKOCS was carried out to identify content of knowledge relevant to collaborative working, develop cloud metadata of knowledge structure from heterogeneous skills, design collaboration moderator service to increase interaction among participants and design knowledge learning services to enhance self-directed learning ability

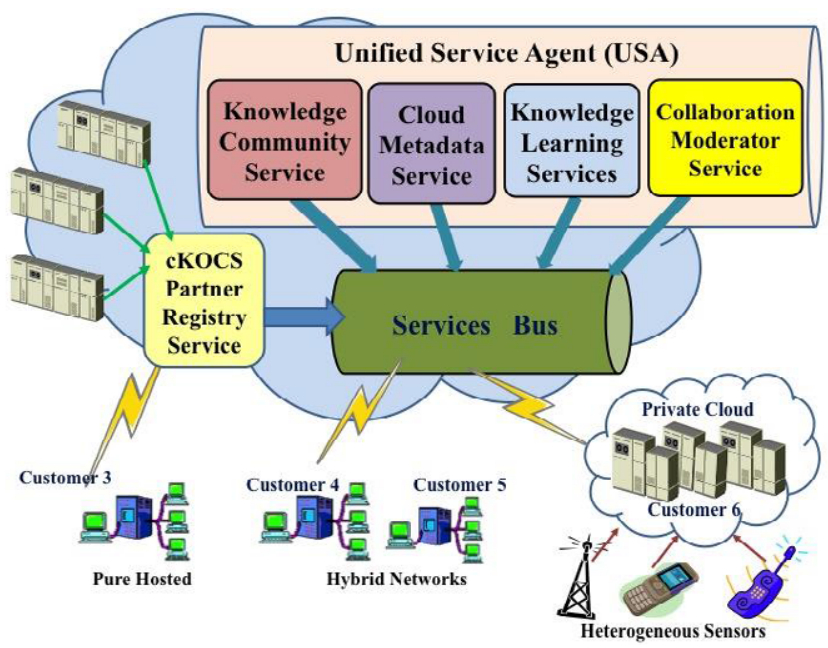

Figure 3: The cKOCS Conceptual Architecture. through the USA for supporting collaborative enterprises decision making. Basically, the USA utilised Azure Cloud Services and Virtual Machines as enabling technologies to facilitate to USA servers' internal communication and interaction between each other more easily. Azure Cloud Services allow using 2 or more web front-end machines (Virtual Machines) to communicate to each other. Moreover, USA was developed as an interoperable service, which can be employed by other services or applications in GCNs cloud environment. As shown in Figure 3, the USA Services includes the Knowledge Community Service, Cloud Metadata Service, Collaboration Moderator Service and Knowledge Learning Services. The functions of each component illustrated as following:

Knowledge community service: In globally distributed collaborative networks, the key to knowledge sharing is the need to know "WHO knows WHAT" or to know "WHOM to contact ABOUT WHAT". There seemed to be a need to know whom to contact about what in this particular organization, something that is far more challenging in globally distributed teams. This organizational aspect, knowing who knows what, has been acknowledged as the key to knowledge sharing activities by several studies [13]. Fulfilling this need will enable the establishment of a knowledge community to be more easily achieved. Therefore, exploitation of a cKOCS interest community will be done directly in order to gain contacts and responses through Knowledge Community Service, which investigates knowledge management activities in communities and networks of practice and the role and use of IT in such knowledge networks. The Knowledge Community Service is a structural framework that facility to capture, classify, store and transfer knowledge to manage correct sharing and communicating of knowledge.

Cloud metadata service: According to Lin and Harding, Harding et al. $[14,15]$, virtual organisation project team members in different parts of the world, each working and using their own preferred terminology, whilst simultaneously sharing information through the mapping mechanisms of the common ontology model. The Cloud Metadata Service developed related work regarding cKOCS ontology model that can be accepted by all participations to make design knowledge effectively accessible across all the project team members. The cKOCS ontology model was implemented by using the Semantic Web technology - the Web Ontology Language (OWL). The Semantic Web relies heavily on formal ontologies to structure data and ontology learning to map between ontologies. In the Cloud Metadata Service, the different enterprises applications (multiple sources / data heterogeneity) are interpreted and connected to the corresponding terminologies through schema matching into the mediated ontology model -cKOCS Ontology Model. Using the Semantic Web Rule Language (SWRL) as a middleware of data conversion rules and provided a mapping logic to automatically derive the transformations from the source attributes to the attributes belonging to the mediated / common format. SWRL is the standard rule language of the semantic web and provides the ability to write Horn-like rules expressed in terms of OWL concepts to reason about OWL individuals. Additionally, the JESS rule engine performs inference.

Collaboration moderator service: Collaboration Moderator Service (CMS) is used to monitor an active project and alerting changes to objects of interest to the formal cKOCS members who might be affected by those changes, establish real-time notification and awareness in collaborative situations, enable ad-hoc collaboration (non-routine request processing) and support business decision-making among members. This is necessary to increase awareness and notification 
Citation: Lin HK, Chen Cl, Wei NC (2015) Enterprise Interoperability for Global Collaborative Networks through Cloud Knowledge-Oriented Collaboration System. J Glob Econ 3: 140. doi:10.4172/2375-4389.1000140

between partners and minimise the risks of detrimental impact to the GCNs of misunderstandings in decisions made by individual partners [16]. The CMS relies on two types of knowledge and information repositories: a knowledge base for storing moderation knowledge and a temporary storage of knowledge and information about processes currently in progress. Two important components of the CMS are knowledge acquisition module (KAM) and the Real Time Module (RTM), which are responsible for interacting with the user for maintaining the moderation knowledge and for real time monitoring activities respectively. The RTM has interfaces for accessing shared collaboration information and to publish/subscribe for events which drive the moderation activities. A high level description of the Collaboration Moderator Service and details of its architecture and implementation can find from Lin and Harding, Swarnkar et al., Harding and Swarnkar $[11,14,17]$

Knowledge learning services: According to Young et al., knowledge management is an approach used not only to detect, capture, organize and store, but also to share, apply knowledge within the business context and to the organisation's benefit. Moreover, the paramount value added by knowledge management to the success of the business is through the reuse of knowledge and in learning from one's own experience, the best practices of others and of past history $[3,4,12]$. The USA Knowledge Learning Services include services to access and reuse repositories of knowledge about generic collaboration patterns and practices, and self-learning knowledge techniques for knowledge acquisition based on Knowledge discovery. Knowledge discovery filter of critical information and patterns from various team members' past and current projects.

\section{Conclusion}

cKOCS empower SMEs enterprises allowing them to significantly accelerate the utilization of collaborative knowledge through the development of appropriate cloud-based tools that provide new opportunities for GCNs communities to share knowledge and experience at the international level.

\section{Reference:}

1. Man-Sze Li, Ricardo C, Guy D, Keith P (2006) Enterprise Interoperability Research Roadmap. Published by European Commission.
2. Cross RL, Roger D Martin, Weiss LM (2006) Mapping the value of employee collaboration. The McKinsey Quarterly 3: 28-41.

3. Popplewell K, Nenad S, Andreas A, Dimitris A, Gregoris M et.al. (2008) Supporting Adaptive Enterprise Collaboration through Semantic Knowledge Services. Enterprise Interoperability III.

4. Harding JA, Popplewell k (2006) Knowledge Reuse and Sharing through Data Mining Manufacturing Data. Industrial Engineering Research Conference, Orlando.

5. Sveiby KE, Roland S (2002) Collaborative climate and effectiveness of knowledge work - an empirical study. Journal of Knowledge Management 6 : 420-433.

6. Kotlarsky J, Oshri I (2005) Social ties, knowledge sharing and successfu collaboration in globally distributed system development projects. European Journal of Information Systems 14: 37-48.

7. Wasko M, Faraj S (2000) It is what one does: why people participate and help others in electronic communities of practice. Journal of Strategic Information Systems 9: 155-173.

8. Thomas JC, Kellogg WA, Erickson T (2001) The knowledge management puzzle: Human and social factors in knowledge management. IBM Systems Journal 40: 863-884

9. Zarb MP (2006) Modelling Participation in Virtual Communities-of-Practice

10. Wulan M, Dai X, Popplewell K (2010) Collaboration Knowledge Ontologies to Support Knowledge Management and Sharing in Virtual Organisations. Enterprise Interoperability IV.

11. Harding JA, Swarnkar R (2013) Implementing collaboration moderator service to support various phases of virtual organisations. International Journal of Production Research 51: 7372-7387.

12. Choudhary AK, Harding JA, Lin HK, Tiwari MK, Shankar R (2011) Knowledge discOvery And daTa minINg inteGrated (KOATING) Moderators for collaborative projects. International journal of production research 49: 7029-7058.

13. James DH, Audris M, Finholt TA, Grinter RE (2000) Distance, dependencies, and delay in a global collaboration. Pennsylvania, United States.

14. Lin HK, Harding JA (2007) A manufacturing system engineering ontology model on the semantic web for inter-enterprise collaboration. Computers in Industry 58: 428-437.

15. Lin HK, Harding JA, Tsai WC (2012) A rule-based knowledge system on semantic web for collaboration moderator services. International Journal of Production Research 50: 805-816

16. Alawamleh M, Popplewell K (2010) Risk Sources Identification in Virtua Organisation". Enterprise Interoperability IV.

17. Swarnkar R, Choudhary AK, Harding JA, Das BP, Young RI (2011) A framework for collaboration moderator services to support knowledge based collaboration. Journal of Intelligent Manufacturing 23: 2003-2023. 